\title{
Note on Wuxi County's Administrative Boundaries, Commercial Districts, and Size
}

In 1724 , Wuxi County was split in two, roughly along a diagonal axis extending from northwest to southeast. The portion incorporating the western and southern districts retained the old name, Wuxi xian (county), while the portion incorporating the northern and eastern districts was called Jinkui xian. At the onset of the Republic in 1912, the two portions were rejoined as Wuxi County. Map 2 in Chapter 6 displays the shape of the Republican-period boundaries; for detailed maps and discussions of Wuxi and Jinkui counties during Qing times, see Zhu I 895, vol. 3. This source estimates that in I 895 , Wuxi had three market towns (zhen); eight townships (xiang) with thirty-three periodic markets (shiji); and four commercial districts just outside the city. Jinkui is estimated to have had ten market towns (zhen); six townships (xiang) with twenty periodic markets (shiji); and one commercial district just north of the city (ibid.: leaves 39-42). In the Republican period, other sources count either sixty-five (Yin I936, I: 29-3 I) or sixty-six zhen (Wuxi xianzhengfu, Wuxi shizheng choubeichu I930: insert map). The discrepancy with the previous numbers can be accounted for if both zhen and shiji subsequently were considered market towns. It is also useful to note that during Republican times in Wuxi, the term shi was used not only to refer to the urban center of the county (as in Wuxi shi [Wuxi City]), but also as a designation for densely settled townships. Other townships were referred to using the more common term, xiang.

Republican-period sources on physical size, cultivated land, and population contain numbers that vary, but the county extended approximately forty miles ( 120 li) from east to west, and thirty-six miles (I I li) from north to south (Wuxi xianzhengfu I935, geography section: I). In I929, one source states that there was approximately $1,255,700$ $\mathrm{mu}$ of cultivated land and a population of 971,800 (Chen 1929: 46-47). Another source estimates that in 1936, Wuxi had a total area of 
I,973,700 $\mathrm{mu}$, cultivated land of $\mathrm{I}, 259,50 \mathrm{I} \mathrm{mu}$, and a total population of $\mathrm{I}, \mathrm{I} 67,926$ (Yin $\mathrm{r} 936, \mathrm{I}: 27-3 \mathrm{I}$ ). It is not entirely clear, but it is possible that the first population figure cited here refers only to those living outside the boundaries of Wuxi shi, the urban core at the center of the county. 